\title{
Report from the NIST-MAS-AMAS Roadmap Workshop on Variable Pressure Scanning Electron Microscopy/Environmental Scanning Electron Microscopy
}

\author{
Scott A. Wight*, Dale E. Newbury* and Brendan Griffin** \\ *National Institute of Standards and Technology, Gaithersburg, MD 20899-8370 \\ ** University of Western Australia, Perth, Australia
}

The National Institute of Standards and Technology (NIST) co-organized and hosted a workshop on Variable Pressure Scanning Electron Microscopy (VPSEM)/Environmental Scanning Electron Microscopy (ESEM) with the Microbeam Analysis Society (MAS) and the Australian Microbeam Analysis Society (AMAS), November 2 to 4, 2005 at the NIST Gaithersburg, Maryland campus. This was the fourth in a series of annual MAS topical conferences jointly sponsored with and held at NIST. The NIST-MAS-AMAS VPSEM-ESEM Roadmap Workshop drew 60 participants from 6 countries and represents the third in a continuing series (1999 and 2001 were hosted by AMAS in Australia) of VPSEM-ESEM workshops. These workshops have brought together leading experts on VPSEM-ESEM to discuss the current state of achievement and understanding and to identify those areas where information is lacking and critical experiments and/or theoretical studies are required to advance the field.

The 2005 VPSEM-ESEM Roadmap Workshop sessions covered six major topics, each of which was highlighted by one or more invited speakers (the speaker only is listed on multiple author presentations):

1. Electron and ion interactions in the gas environment (including detectors)

Ion Dynamics in the ESEM

Electron-Gas scattering processes in low vacuum

SE Detector Design Criteria for different low vacuum applications

Gas-surface interactions in ESEM

2. Contrast mechanisms in VPSEM-ESEM

Introduction - what do we mean by contrast?

Contrast in Charge Collection Microscopy, how, why, what?

CCI Contrast from soft tissue sample

Microscopy and Microanalysis in liquids

Atomic level imaging in liquids

3. VP Metrology

Special Considerations for CD-Metrology in Low Vacuum

The effects of operating parameters on environmental SEM Metrology

Optimizing signal to noise ratio in gas cascade amplification

ESEM-based Reference Metrology System

ESEM charge control and contamination reduction for high resolution

critical dimension microscopy

4. Dynamic Experiments

Dynamic Experiments in Heating and Cooling in the ESEM

ESEM experiments in a gas flow heating stage

5. VPSEM-ESEM FIB
Matthew Phillips

Brad Thiel

Ralph Knowles

Milos Toth

David Joy

Brendan Griffin

Peda Clode

David Joy

Niels de Jonge

Brad Thiel

David Joy

Vasiliki Tileli

Andras Vladar

Milos Toth

Ken Severin

Charlotte Appel 
FIB/ESEM DualBeam Instrumentation and Applications An ESEM and FIB Workstation in one Instrument - Applications

FIB SEM of Insulating Materials: Issues, Solutions, Questions 6. Microanalysis in VPSEM-ESEM

Quantitative Analysis Procedures

X-ray Optics for Reduction of Gas Scattering Contribution

Accuracy and Precision of Quantitative X-ray Analysis using ESEM Monte Carlo Software for Estimating Gas Scatter Contributions
Lucille Giannuzzi

John Mansfield

Debbie Stokes

Eric Doehne

Walter Gibson

Robert Carlton

Nicholas Ritchie

The seventh session comprised the Roadmap discussions, covering a wide range of topics organized around a series of provocative statements with designated pro and con responders:

1. We should still separate VPSEM $(<270 \mathrm{~Pa})$ from ESEM ( $>2$ Torr $)$

2. True high resolution SEM metrology is only possible with VP-ESEM

3. $90 \%$ of SEM will be VP-ESEM

4. Secondary electron imaging does not occur in VP-ESEM - it is all tertiary

5. CCI provide images of sample detail that is unique to the VP-ESEM

6. Charge implantation/accumulation in insulators dominate imaging and analysis processes in VP-ESEM

7. Ion capture by widgets is useful and should be very near the detector

8. Self-cleaning processes in VP-ESEM are critical to semiconductor applications

9. Luminescence-based detectors are more efficient and will become the detector of choice in VP-ESEM

10. $\mathrm{S} / \mathrm{N}$ is be the prime measure of VP-ESEM detector performance

11. Field emission sources restrict imaging gas options by excluding noble gases

12. X-ray microanalysis in the VP-ESEM must be considered macroanalysis

13. Reliable, routine quantitative microanalysis of insulators is impossible in VP-ESEM

14. Quantomix capsules are a better approach for wet imaging \& microanalysis

An attempt has been made to capture as much as practically possible of the information presented at the workshop and make it available to a worldwide audience. Session speakers, roadmap responders, and participants were therefore asked to provide text and/or visual records of their presentations and written comments. The record is available at http://www.microbeamanalysis.org/mas/workshop/CD.htm

While such a Web-based resource can never replace the experience of actually attending the Workshop, the material may still prove useful to the VPSEM-ESEM community as a source of current thinking and information on current projects, made available substantially prior to the appearance of the finished material in the appropriate journals. 